\title{
Feasible combinatorial matrix theory
}

\author{
Polytime proofs for König's Min-Max and related theorems
}

\author{
Ariel Fernández \\ McMaster University \\ Hamilton, Canada \\ fernanag@mcmaster.ca
}

\author{
Michael Soltys \\ McMaster University \\ Hamilton, Canada \\ soltys@mcmaster.ca
}

\begin{abstract}
We show that the well-known König's Min-Max Theorem (KMM), a fundamental result in combinatorial matrix theory, can be proven in the first order theory LA with induction restricted to $\Sigma_{1}^{B}$ formulas. This is an improvement over the standard textbook proof of KMM which requires $\Pi_{2}^{B}$ induction, and hence does not yield feasible proofs - while our new approach does. LA is a weak theory that essentially captures the ring properties of matrices; however, equipped with $\Sigma_{1}^{B}$ induction LA is capable of proving KMM, and a host of other combinatorial properties such as Menger's, Hall's and Dilworth's Theorems. Therefore, our result formalizes Min-Max type of reasoning within a feasible framework.
\end{abstract}

\section{INTRODUCTION}

In this paper we are concerned with the complexity of formalizing reasoning about combinatorial matrix theory. We are interested in the strength of the bounded arithmetic theories necessary in order to prove the fundamental results of this field. We show, by introducing new proof techniques, that the logical theory LA with induction restricted to bounded existential matrix quantification is sufficient to formalize a large portion of combinatorial matrix theory.

Perhaps the most famous theorem in combinatorial matrix theory is the König's Mini-Max Theorem (KMM) which arises naturally in all areas of combinatorial algorithms for example "network flows" with "min-cut max-flow" type of reasoning. See [1], [2] for the original papers introducing $\mathrm{KMM}$, and see [3] for recent work related to formalizing proof of correctness of the Hungarian algorithm, which is an algorithm based on KMM. As far as we know, we give the first feasible proof of KMM.

As KMM is a cornerstone result, it has several counterparts in related areas of mathematics: Menger's Theorem, counting disjoint paths; Hall's Theorem, giving necessary and sufficient conditions for the existence of a "system of distinct representatives" of a collection of sets; Dilworth's Theorem, counting the number of disjoint chains in a poset, etc. We note that we actually show the equivalence of KMM with a restricted version of Menger's Theorem.

We show that KMM can be proven feasibly (Theorem 11, and we do so with a new proof of KMM that relies on introducing a new notion (Definition 3). Furthermore, we show that the theorems related to KMM, and listed in the above paragraph, can also be proven feasibly; in fact, all these theorems are equivalent to KMM, and the equivalence can be shown in LA (Theorem 9). We believe that this captures the proof complexity of Min-Max reasoning.

Our results show that Min-Max reasoning can be formalized with uniform Extended Frege. It would be very interesting to know whether the techniques recently introduced by [4] could bring the complexity further down to quasi-polynomial Frege.

\section{BACKGROUND}

KMM states the following: Let $A$ be a matrix of size $n \times m$ with entries in $\{0,1\}$, what we sometimes call a 0-1 matrix. A line of $A$ is an entire row or column of $A$; given an entry $A_{i j}$ of $A$ (when giving LA formulas we shall denote such an entry with $A(i, j)$ ), we say that a line covers that entry if this line is either row $i$ or column $j$. Then, the minimal number of lines in $A$ that cover all of the $1 \mathrm{~s}$ in $A$ is equal to the maximal number of $1 \mathrm{~s}$ in $A$ with no two of the $1 \mathrm{~s}$ on the same line. Note that KMM is stated for $n \times m$ matrices, but for simplicity we shall state it for $n \times n$ (i.e., square) matrices; of course, all results given in this paper hold for both.

See [5, pg. 6] for a classical discussion and proof of KMM (and note that the proof relies, implicitly, on a $\Pi_{2}^{B}$ type of induction).

We give a feasible proof of KMM in the logical theory LA defined in [6]. By restricting the induction to be over $\Sigma_{1}^{B}$ formulas, that is formulas whose prenex form consists of a block of bounded existential matrix quantifiers - and no other matrix quantifiers - we manage to prove KMM in a fragment of $\mathbf{L A}$ called $\exists \mathbf{L A}$. While the matrices in the statements of the theorems have $\{0,1\}$ entries, we assume that the underlying ring is $\mathbb{Z}$, the set of integers. We require the integers as one of our fundamental operations will be counting the number of $1 \mathrm{~s}$ in a 0-1 matrix, i.e., computing $\Sigma A$, the sum of all the entries of $A$.

The background for $\mathbf{L A}$ is given in a one-page Appendix at the end of the paper, but the interested reader can read the full treatment in [6]. We shall leave routine details of proofs to the reader, in the interest of space.

The main contribution of this paper is to show that KMM can be proven in the theory $\exists \mathbf{L A}$ which implies that it can be proven feasibly. We mention here an important observation of Jeřábek from [7, pg. 44]: $\exists \mathbf{L A}$ does not necessarily translate into a polytime proof system (e.g., extended Frege) when the matrices are over $\mathbb{Z}$. However, if we restrict the quantified 
matrices to be over $\{0,1\}$, which is what we do in our proofs, it readily translates into extended Frege.

We use $|A| \leq n$ to abbreviate $r(A) \leq n \wedge c(A) \leq n$, that is, the number of rows of $A$ is bounded by $n$, and the number of columns of $A$ is bounded by $n$. We let $(\exists A \leq n) \alpha-$ resp. $(\forall A \leq n) \alpha$ - abbreviate $(\exists A)[|A| \leq n \wedge \alpha]$ - resp. $(\forall A)[|A| \leq n \rightarrow \alpha]$. These are bounded matrix quantifiers.

Note that LA allows for reasoning with arbitrary quantification; however, in $\mathbf{L A}$ we only allow induction over formulas without matrix quantification. On the other hand, in $\exists \mathbf{L A}$ we allow induction over so called $\Sigma_{1}^{B}$ formulas. These are formulas, which when presented in prenex form, contain a single block of bounded existential matrix quantifiers. The set of formulas $\Pi_{1}^{B}$ is defined similarly, except the block of quantifiers is universal.

In general, $\Sigma_{i}^{B}$ is the set of formulas which, when presented in prenex form, start with a block of bounded existential matrix quantifiers, followed by a block of bounded universal matrix quantifiers, with $i$ such alternating blocks. The set $\Pi_{i}^{B}$ is the same, except it starts with a block of universal matrix quantifiers.

The main contribution of our paper can now be stated more precisely: following König's original proof of KMM, which is also the standard presentation of the proof in the literature (see the seminal work in the field [5, pg. 6]) one can construct a proof with $\Pi_{2}^{B}$ induction, which does not yield translations into extended Frege proofs. On the other hand, we are able to give a proof that uses only $\Sigma_{1}^{B}$ induction, which do yield extended Frege proofs, and thereby a feasible proof of KMM. Our insight is that while we are doing induction over the size of matrices, we can pre-arrange our matrices in a way that lowers the complexity of the induction. This is accomplished with the procedure outlined in the Definition 3 - the diagonal property for matrices - and the subsequent proof of Claim 4

We show how to express the concepts necessary to state KMM in the language $\mathcal{L}_{\mathbf{L A}}$. First, we say that the matrix $\alpha$ is a cover of a matrix $A$ with the predicate:

$$
\begin{aligned}
& \operatorname{Cover}(A, \alpha):= \\
& \forall i, j \leq r(A)(A(i, j)=1 \rightarrow \alpha(1, i)=1 \vee \alpha(2, j)=1)
\end{aligned}
$$

We allow some leeway with notation: $\forall i, j \leq r(A)$ is of course $(\forall i \leq r(A))(\forall j \leq r(A))$. The matrix $\alpha$ keeps track of the lines that cover $A$; it does so with two rows: the top row keeps track of the horizontal lines, and the bottom row keeps track of the vertical line. The condition ensures that any 1 in $A$ is covered by some line stipulated in $\alpha$.

The next predicate expresses that the matrix $\beta$ is a selection of 1 s of $A$ so that no two of these lines are on the same line. Thus, $\beta$ can be seen as a "subset" of a permutation matrix; that is, each $\beta$ is obtained from some permutation matrix by deleting some (possibly none) of the $1 \mathrm{~s}$. We say that $\beta$ is a selection of $A$, and it is given with the following formula:

$$
\begin{gathered}
\operatorname{Select}(A, \beta):=\forall i, j \leq r(A)((\beta(i, j)=1 \rightarrow A(i, j)=1) \\
\wedge \forall k \leq r(A)(\beta(i, j)=1 \rightarrow \beta(i, k)=0 \wedge \beta(k, j)=0))
\end{gathered}
$$

We are interested in a minimum cover (as few $1 \mathrm{~s}$ in $\alpha$ as possible) and a maximum selection (as many $1 \mathrm{~s}$ in $\beta$ as possible). The following two predicates express that $\alpha$ is a minimum cover and $\beta$ a maximum selection.

$$
\begin{aligned}
& \operatorname{Min} \operatorname{Cover}(A, \alpha):= \\
& \operatorname{Cover}(A, \alpha) \wedge \forall \alpha^{\prime} \leq c(\alpha)\left(\operatorname{Cover}\left(A, \alpha^{\prime}\right) \rightarrow \Sigma \alpha^{\prime} \geq \Sigma \alpha\right) \\
& \operatorname{MaxSelect}(A, \beta):= \\
& \operatorname{Select}(A, \beta) \wedge \forall \beta^{\prime} \leq r(\beta)\left(\operatorname{Select}\left(A, \beta^{\prime}\right) \rightarrow \Sigma \beta^{\prime} \leq \Sigma \beta\right)
\end{aligned}
$$

Clearly MinCover and MaxSelect are $\Pi_{1}^{B}$ formulas. We can now state $\mathrm{KMM}$ in the language of $\mathcal{L}_{\mathbf{L} \mathbf{A}}$ as follows:

$$
\operatorname{MinCover}(A, \alpha) \wedge \operatorname{MaxSelect}(A, \beta) \rightarrow \Sigma \alpha=\Sigma \beta
$$

Note that (5) is a $\Sigma_{1}^{B}$ formula. The reason is that in prenex form, the universal matrix quantifiers in MinCover and MaxSelect become existential as we pull them out of the implication; they are also bounded.

Let $\operatorname{KMM}(A, n)$ be the following $\Sigma_{1}^{B}$ formula: it is a conjunction of the statement that $A$ is an $n \times n$ matrix, which we abbreviate informally as $|A|=n$, and which in $\mathcal{L}_{\mathbf{L A}}$ is stated as $r(A)=n \wedge c(A)=n$, and (5) in prenex form:

$$
\operatorname{KMM}(A, n):=|A|=n \wedge \operatorname{prenex}(\underline{5}) .
$$

Given a matrix $A$, let $l_{A}$ and $o_{A}$ denote the minimum number of lines necessary to cover all the $1 \mathrm{~s}$ of $A$, and the maximum number of $1 \mathrm{~s}$ no two on the same line, respectively. (Of course, König's theorem says that for all $A$, $l_{A}=o_{A}$.) In terms of the definitions just given, we have that $l_{A}=\Sigma \alpha$ where $\operatorname{Min} \operatorname{Cover}(A, \alpha)$, and $o_{A}=\Sigma \beta$ where $\operatorname{MaxSelect}(A, \beta)$.

Finally, the fact that $P$ is a permutation matrix can be stated easily with a predicate free of matrix quantification; see, for example, [8].

\section{THE MAIN RESULT}

With the basic machinery in place, we can now prove the main Theorem of the paper.

Theorem 1: $\exists \mathbf{L A} \vdash \mathrm{KMM}$.

What this Theorem says is that KMM can be shown in LA with $\Sigma_{1}^{B}$ induction, and thus in uniform extended Frege, which in turn means feasibly. The rest of this section consists in a proof of this theorem.

Some of the intermediate results can be shown with just LA induction (i.e., induction over formulas without matrix quantifiers, that is, over formulas in $\Sigma_{0}^{B}=\Pi_{0}^{B}$ ). We use the weaker theory whenever possible.

Lemma 2: Given a matrix $A$, and given any permutation matrix $P$, we have

$$
\begin{aligned}
& \text { - } \mathbf{L A} \vdash l_{P A}=l_{A P}=l_{A} \\
& \text { - } \mathbf{L A} \vdash o_{P A}=o_{A P}=o_{A}
\end{aligned}
$$

That is, these four equalities can be proven in $\mathbf{L A}$, i.e., with induction restricted to formulas without matrix quantifiers.

Proof: LA shows that if we reorder the rows or columns (or both) of a given matrix $A$, then the new matrix, call it $A^{\prime}$, where $A^{\prime}=P A$ or $A^{\prime}=A P$, has the same size minimum 
cover and the same size maximum selection. Of course, we can reorder both rows and columns by applying the statement twice: $A^{\prime}=P A$ and $A^{\prime \prime}=A^{\prime} Q=P A Q$.

The first thing that we need to show is that:

- $\mathbf{L A} \vdash \operatorname{Cover}(A, \alpha) \rightarrow \operatorname{Cover}\left(A^{\prime}, \alpha^{\prime}\right)$

- $\mathbf{L A} \vdash \operatorname{Select}(A, \beta) \rightarrow \operatorname{Select}\left(A^{\prime}, \beta^{\prime}\right)$

where $A^{\prime}$ is defined as in the above paragraph, and $\alpha^{\prime}$ is the same as $\alpha$, except the first row of $\alpha$ is now reordered by the same permutation $P$ that multiplied $A$ on the left (and the second row of $\alpha$ is reordered if $P$ multiplied $A$ on the right). The matrix $\beta$ is even easier to compute, as $\beta^{\prime}=P \beta$ if $A^{\prime}=P A$, and $\beta^{\prime}=\beta P$ if $A^{\prime}=A P$. It follows from $P$ being a permutation matrix that $\Sigma \alpha=\Sigma \alpha^{\prime}$ and $\Sigma \beta=\Sigma \beta^{\prime}$ : we can show by LA induction on the size of matrices that if $X^{\prime}$ is the result of rearranging $X$ (i.e., $X^{\prime}=P X Q$, where $P, Q$ are permutation matrices), then $\Sigma X=\Sigma X^{\prime}$. We do so first on $X$ consisting of a single row, by induction on the length of the row. Then we take the single row as the basis case for induction over the number of rows of a general $X$.

It is clear that given $A^{\prime}$, the cover $\alpha^{\prime}$ has been adjusted appropriately; same for the selection $\beta^{\prime}$. We can prove it formally in $\mathbf{L A}$ by contradiction: suppose some 1 in $A^{\prime}$ is not covered in $\alpha^{\prime}$; then the same 1 in $A$ would not be covered by $\alpha$. For the selections, note that reordering rows and/or columns we maintain the property of being a selection: we can again prove this formally in LA by contradiction: if $\beta^{\prime}$ has two $1 \mathrm{~s}$ on the same line, then so would $\beta$.

The next thing to show is that

- $\mathbf{L A} \vdash \operatorname{Min} \operatorname{Cover}(A, \alpha) \rightarrow \operatorname{Min} \operatorname{Cover}\left(A^{\prime}, \alpha^{\prime}\right)$

- $\mathbf{L A} \vdash \operatorname{MaxSelect}(A, \beta) \rightarrow \operatorname{MaxSelect}\left(A^{\prime}, \beta^{\prime}\right)$

and the reasoning that accomplishes this is by contradiction. As permuting only reorders the matrices (it does not add or take away 1s), if the right-hand side does not hold, we would get that the left-hand side does not hold by applying the inverse of the permutation matrix.

All these arguments can be easily formalized in $\mathbf{L A}$, and we leave the details to the reader.

The next definition is a key concept in the $\Sigma_{1}^{B}$ proof of KMM

Definition 3: We say that an $n \times n$ matrix over $\{0,1\}$ has the diagonal property if for each diagonal entry $(i, i)$ of $A$, either $A_{i i}=1$, or $(\forall j \geq i)\left[A_{i j}=0 \wedge A_{j i}=0\right]$.

Claim 4: Given any matrix $A, \exists \mathbf{L} \mathbf{A}$ proves that there exist permutation matrices $P, Q$ such that $P A Q$ has the diagonal property.

Proof: We construct $P, Q$ inductively on $n=|A|$. Let the $i$-th layer of $A$ consist of the following entries of $A$ : $A_{i j}$, for $j=i, \ldots, n$ and $A_{j i}$ for $j=i+1, \ldots, n$. Thus, the first layer consists of the first row and column of $A$, and the $n$-th layer (also the last layer), is just $A_{n n}$. We transform $A$ by layers, $i=1,2,3, \ldots$. At step $i$, let $A^{\prime}$ be the result of having dealt already with the first $i-1$ layers. If $A_{i i}^{\prime}=1$ move to the next layer, $i+1$. Otherwise, find a 1 in layer $i$ of $A^{\prime}$. If there is no 1 , also move on to the next layer, $i+1$. If there is a 1 , permute it from position $A_{i j^{\prime}}, j^{\prime} \in\{i, \ldots, n\}$ to $A_{i i}^{\prime}$, or from position
$A_{j^{\prime} i}, j^{\prime} \in\{i+1, \ldots, n\}$. Note that such a permutation does not disturb the work done in the previous layers; that is, if $A_{k k}^{\prime}, k<i$, was a 1 , it continues being a 1 , and if it was not a 1 , then there are no $1 \mathrm{~s}$ in layer $k$ of $A^{\prime}$.

It is Claim 4 that allows us to bring down the complexity of the proof of KMM from $\Pi_{2}^{B}$ to $\Sigma_{1}^{B}$. As we shall see, by transforming $A$ into $A^{\prime}$, so that $A^{\prime}=P A Q$ where $P, Q$ are permutation matrices and $A^{\prime}$ has the diagonal form, we can prove KMM for $A^{\prime}$ with just $\Sigma_{1}^{B}$ induction, and then by Lemma 2 we obtain an $\exists \mathbf{L A}$ proof of KMM for $A$. All of this is made precise in the following Lemma; recall that $\operatorname{KMM}(A, n)$ is defined in (6).

Lemma 5: $\exists \mathbf{L A} \vdash \forall n \operatorname{KMM}(A, n)$.

We are going to prove Lemma 5 by induction on $n$, breaking it down into Claims 6 and 7 . Once we have that $\forall n \operatorname{KMM}(A, n)$, we replace $n$ with $|A|$, and obtain an $\exists \mathbf{L A}$ proof of (5), and thereby a proof of Theorem 1 .

From Claims 2 and 4 we know that it is sufficient to prove Lemma 5 for appropriate $P A Q$, which ensures the diagonal property spelled out in Claim 4. Thus, in order to simplify notation, we assume that our $A$ is the result of applying the permutations; i.e., $A$ has the diagonal property.

Claim 6: $\mathbf{L A} \vdash o_{A} \leq l_{A}$.

Proof: Given a covering of $A$ consisting of $l_{A}$ lines, we know that every 1 we pick for a maximal selection of $1 \mathrm{~s}$ has to be on one of the lines of the covering. We also know that we cannot pick more than one 1 from each line. Thus, the number of lines in the covering provide an upper bound on the size of such selection, giving us $o_{A} \leq l_{A}$.

We can formalize this argument in LA as follows: let $A^{\prime}$ be a matrix whose rows represent the $l_{A}$ lines of a covering, and whose columns represent the $o_{A} 1 \mathrm{~s}$ no two on the same line. Let $A^{\prime}(i, j)=1 \Longleftrightarrow$ the line labeled with $i$ covers the 1 labeled with $j$. Then,

$$
\begin{aligned}
o_{A} & =c\left(A^{\prime}\right) \leq \Sigma A^{\prime} \\
& =\Sigma_{i}\left(\Sigma \lambda p q\left\langle 1, c\left(A^{\prime}\right), A^{\prime}(i, q)\right\rangle\right) \\
& \leq \Sigma_{i} 1=r\left(A^{\prime}\right)=l_{A},
\end{aligned}
$$

where the inequality in the line labeled by $(*)$ can be shown by induction on the number of columns of a matrix which has the condition that each column contains at least one 1; and the equality labeled with $(* *)$ follows from the fact that we can add all the entries in a matrix by rows (and $A^{\prime}$ is such that each row contains at most one 1).

We briefly discuss the implications of Claim 6 for the provability of variants of the pigeonhole principle (PHP) in LA in Section VI.

As Claim 6 shows, LA is sufficient to prove $o_{A} \leq l_{A}$; on the other hand, we seem to require the stronger theory $\exists \mathbf{L A}$ (which is $\mathbf{L A}$ with induction over $\Sigma_{1}^{B}$ formulas) in order to prove the other direction of the inequality.

Claim 7: $\exists \mathbf{L A} \vdash o_{A} \geq l_{A}$.

Proof: By induction on $n=|A|$. We assume throughout the proof that the matrix has the diagonal property (see 
Definition 3). Let

$$
A=\left[\begin{array}{l|l}
a & R \\
\hline S & M
\end{array}\right],
$$

where $a$ is the top-left entry, and $M$ the principal sub-matrix of $A$, and $R$ (resp. $S$ ) is $1 \times(n-1)$ (resp. $(n-1) \times 1)$. From the diagonal property we know that one of the following two cases is true:

Case 1. $a=1$

Case 2. $a, R, S$ consist entirely of zeros

In the second case, $o_{A} \geq l_{A}$ follows directly from the induction hypothesis, $o_{M} \geq l_{M}$, as $o_{A}=o_{M} \geq l_{M}=l_{A}$. Thus, it is the first case, $a=1$, that is interesting. The first case, in turn, can be broken up into two subcases: $l_{M}=n-1$ and $l_{M}<n-1$.

Subcase (1-a) $l_{M}=n-1$

By induction hypothesis, $o_{M} \geq l_{M}=n-1$. We also have that $a=1$, and $a$ is in position $(1,1)$, and hence no matter what subset of $1 \mathrm{~s}$ is selected from $M$, none of them lie on the same line as $a$. Therefore, $o_{A} \geq o_{M}+1$. Since $o_{M} \geq n-1$, $o_{A} \geq n$, and since we can always cover $A$ with $n$ lines, we have that $n \geq l_{A}$, and so $o_{A} \geq l_{A}$.

Subcase (1-b) $l_{M}<n-1$

Consider a covering of $M$ of size $l_{M}<n-1$. We break this case down into two further sub-subcases, depending on whether this particular covering has, or has not, the following property: when all lines of the covering (of $M$ ) are extended to the entire matrix $A$, they cover all the $1 \mathrm{~s}$ in $S$, or they cover all the $1 \mathrm{~s}$ in $R$.

For the sake of formalizing the proof in $\mathbf{L A}$, we define the notion of "extension" more precisely.

Definition 8: Let $A$ and $M$ be as in (7), and let $C_{M}$ be a set of lines of $M$, i.e., $C_{M}$ consists of rows $i_{1}, i_{2}, \ldots, i_{k}$, and columns $j_{1}, j_{2}, \ldots, j_{\ell}$. The extension of $C_{M}$ to $C_{A}$ is simply the set of rows $i_{1}+1, i_{2}+1, \ldots, i_{k}+1$, and the set of columns $j_{1}+1, j_{2}+1, \ldots, j_{\ell}+1$.

Sub-subcase (1-b-i) The given covering of $M$ of size $l_{M}$, when extended to the full matrix $A$, covers all the $1 \mathrm{~s}$ in $S$, or covers all the $1 \mathrm{~s}$ in $R$ (or possibly both).

By induction hypothesis, $o_{M}=l_{M}$, and so we can pick $l_{M}$ $1 \mathrm{~s}$ in $M$, no two of them on the same line, plus $a$, to have a selection of $1 \mathrm{~s}$, no two on the same line, of size $l_{M}+1$. Thus, $o_{A} \geq l_{M}+1$. On the other hand, there is a covering of $A$ consisting of the lines covering $M$ (now extended to all of $A$ ), plus the first column of $A$ (if it was $R$ that was fully covered by the extension), or the first row of $A$ (if it was $S$ that was fully covered by the extension). Note that if both $R, S$ were fully covered by the extension, just pick arbitrarily the first row or the first column of $A$, as all that matters in this case is to cover $a=1$. Therefore, $l_{M}+1 \geq l_{A}$, and so $o_{A} \geq l_{A}$.

Sub-subcase (1-b-ii) The given covering of $M$, of size $l_{M}$, when extended to $A$, it leaves some 1 in $R$ uncovered, and some 1 in $S$ uncovered.
In that case, $o_{A} \geq o_{M}+2$, where we picked a covering of $M$, extended it to $A$, and picked a selection of $1 \mathrm{~s}$ of size $o_{M}$, no two on the same line, plus a 1 in $R$ uncovered by the extension, and a 1 in $S$ uncovered by the extension, to create a selection of $1 \mathrm{~s}$ in $A$ of size $o_{M}+2$, no two on the same line. On the other hand, $l_{M}+2$ lines cover all of $A$ : the extension of the cover of $M$ of size $l_{M}$ plus the first row and first column of $A$. Thus $o_{M}+2=l_{M}+2 \geq l_{A}$. Altogether, $o_{A} \geq l_{A}$.

This ends the proof of Claim 7

\section{INDUCED ALGORITHM}

The standard KMM Theorem is stated as an implication (see (5)), and hence it makes no assertions about the actual existence of a minimal covering or maximal selection of $1 \mathrm{~s}$, let alone how to compute them. It only says that if they do exist, they are equal. However, the proof of Lemma 5 suggests an algorithm for computing both.

Note that computing a minimal cover can be accomplished in polytime with the well-known Karp-Hopcroft (KH) algorithm (see [9]) as follows: First use the $\mathrm{KH}$ algorithm to compute a "maximal matching," which in this case is simply a maximal selection of $1 \mathrm{~s}$ (when we view $A-$ in the natural way - as the adjacency matrix of a bipartite graph). In [10], the authors show how to convert, in linear time, a maximal selection into a minimal cover.

Certainly the correctness of the algorithms mentioned in the above paragraph can be shown in $\exists \mathbf{L A}$ (as it captures polytime reasoning — see [6]), and so it follows that we can prove in $\exists \mathbf{L A}$ the existence of a minimal cover and maximum selection. Therefore, $\exists \mathbf{L A}$ can prove something stronger than (5). Namely, it can not only show that if we have a minimal cover and a maximal selection, then they have the same size, but rather, that there always exists a minimal cover and maximal selection, and the two are of equal size.

However, instead of doing the heavy lifting necessary to formalize the correctness of $\mathrm{HK}$ and [10] in $\exists \mathbf{L A}$, we present a new simple polytime algorithm for computing minimal covers based on the proof of Lemma 5. Note that a similar argument would show the existence of a polytime algorithm for maximal selection - we leave that to the reader.

The algorithm works as follows: given a $0-1$ matrix $A$, we first put $A$ in the diagonal form (see Definition 3). We now work with $A$ which is assumed to be in diagonal form and proceed by computing recursively $l_{M}$, the size of a minimal cover of $M$, where $M$ is the principal submatrix of $A$. Keeping in mind the form of $A$ given by (7), we have the following cases:

Case 1. If $a=0$ (in which case $R, S$ are also zero, by the fact that $A$ has been put in diagonal form), then $l_{A}=l_{M}$, and proceed to compute the minimal cover $C$ of $M$; output $C^{\prime}$, the extension of $C$ (see Definition 8 ).

Case 2. If $a \neq 0$, we first examine $R$ to see if the matrix $M^{\prime}$, consisting of the columns of $M$ minus those columns of $M$ which correspond to $1 \mathrm{~s}$ in $R$, has a cover of size $l_{M}-\Sigma R$ (of course, if $l_{M}<\Sigma R$, then the answer is "no"). 
If the answer is "yes", compute the minimal cover of $M^{\prime}$, $C_{M^{\prime}}$. Then let $C_{M}$ be the cover of $M$ consisting of the lines in $C_{M^{\prime}}$ properly renamed to account for the deletion of columns that transformed $M$ into $M^{\prime}$, plus the columns of $M$ corresponding the the $1 \mathrm{~s}$ in $R$. Then, $C_{A}$ is the result of extending $C_{M}$ and adding the first column of $A$.

If the answer is "no", repeat the same with $S$ : let $M^{\prime}$ be the result of subtracting from $M$ the rows corresponding to the rows with $1 \mathrm{~s}$ in $S$. Check whether $M^{\prime}$ has a cover of size $l_{M}-\Sigma S$. If the answer is "yes" then build a cover for $A$ as in the $R$-case.

If the answer is "no", then compute any minimal cover for $M$, extend it to $A$, and add the first row and column of $A$; this results in a cover for $A$.

At the end, we apply the permutations $P, Q$ that converted $A$ to the diagonal form, to the final $C$, and output that as the minimal cover of the original $A$. As was mentioned above, a similar polytime recursive algorithm can compute a maximal selection of $1 \mathrm{~s}$; we leave that to the reader.

\section{RELATED THEOREMS}

In this section we are going to prove that the various reformulations of KMM, arising in graph theory and partial orders, can be proven equivalent to KMM in low complexity (LA), and therefore they also have feasible proofs. We state this as the following theorem:

Theorem 9: The theory $\mathbf{L A}$ proves the equivalence of KMM, Menger's (restricted), Hall's and Dilworth's Theorems.

The proof of consists of Lemmas 11 and 13 , showing the equivalence of KMM and a restricted version of Menger's Theorem in Subsection A; Lemmas 15 and 16, showing the equivalence of KMM and Hall's Theorem in Subsection B; Lemmas 17 and 18, showing the equivalence of KMM and Dilworth's Theorem in Subsection C. Each subsection starts with a description of how to formalize the given Theorem, followed by the two Lemmas giving the two directions of the equivalence.

\section{A. Menger's Theorem}

Given a graph $G=(V, E)$, an $x, y$-path in $G$ is a sequence of distinct vertices $v_{1}, v_{2}, \ldots, v_{n}$ such that $x=v_{1}$ and $y=v_{n}$ and for all $1 \leq i<n,\left(v_{i}, v_{i+1}\right) \in E$. The vertices $\left\{v_{2}, \ldots, v_{n-1}\right\}$ are called internal vertices; we say that two $x, y$-paths are internally disjoint if they do not have internal vertices in common.

Given two distinct vertices $x, y \in V$, we say that $S \subseteq E$ is an $x, y$-cut if there is no path from $x$ to $y$ in the graph $G^{\prime}=(V, E-S)$. Let $\kappa(x, y)$ represent the size of the smallest $x, y$-cut, and let $\lambda(x, y)$ represent the size of the largest set of pairwise internally disjoint $x, y$-paths.

Menger's theorem states that for any graph $G=(V, E)$, if $x, y \in V$ and $(x, y) \notin E$, then the minimum size of an $x, y$-cut equals the maximum number of pairwise internally disjoint $x, y$-paths. That is, $\kappa(x, y)=\lambda(x, y)$. For more details on Menger's Theorem turn to [11], [12], [13]. Menger's Theorem is of course the familiar Min-Cut Max-Flow Theorem where all edges have capacity 1 .

As was noted earlier, we do not show the equivalence of KMM with the standard version of Menger's Theorem, but rather with a restricted version. Since this restriction is important, we give it in a definition.

Definition 10: Given a graph $G=(V, E)$, we say that a pair of vertices $x, y \in V$ is restricted if any $x, y$-path shares edges with at most one other $x, y$-path.

The intuition behind this definition is that given an $x, y$ restricted pair, there is little "redundancy" in the paths between $x$ and $y$.

We now show how to state Menger's theorem in $\mathcal{L}_{\mathbf{L}} \mathbf{A}$. We start by defining the $\Sigma_{0}^{B}$ predicate $\operatorname{Path}(A, x, y, \alpha)$, which states that $\alpha$ encodes the internal vertices of a path from $x$ to $y$ in $A$. We define Path by parts; first we state that $\alpha$ has at most one 1 in each row and column:

$$
\begin{gathered}
(\forall l \leq n-2)\left[\Sigma \lambda_{i j}\langle 1, n-2, \alpha(l, j)\rangle=1\right. \\
\left.\wedge \Sigma \lambda_{i j}\langle n-2,1, \alpha(i, l)\rangle=1\right]
\end{gathered}
$$

Then we say that if the $l$-th node is $p$ and $l+1$-th node is $q$, then there is an edge between $p$ and $q$ :

$$
\begin{aligned}
& (\forall l, p, q \leq n-3) \\
& \quad(\alpha(l, p)=1 \wedge \alpha(l+1, q)=1) \rightarrow A(p, q)=1
\end{aligned}
$$

Note that in general different paths are of different lengths; this can be dealt with in a number of ways: for example, by padding $\alpha$ with repetitions of the last row (so that each $\alpha$ has exactly $n-2$ rows). We assume that this is what we do, and the reader can check that $\mathcal{L}_{\mathbf{L A}}$ can express this easily.

If $i$ is the first intermediate node then $(x, i) \in E$, and if $i$ is the last intermediate node then $(i, y) \in E$ :

$$
\begin{aligned}
& \alpha(1, i)=1 \rightarrow A(x, i)=1 \\
& \wedge \alpha(n-2, i)=1 \rightarrow A(i, y)=1
\end{aligned}
$$

Putting it all together, the $\Sigma_{0}^{B}$ formula expressing Path is given by the conjunction of $A(x, y)=0$ together with the above properties, i.e.,

$$
\operatorname{Path}(A, x, y, \alpha):=(8) \wedge(9) \wedge(10) \wedge A(x, y)=0 .
$$

Finally, we state that two paths $\alpha, \alpha^{\prime}$ are internally disjoint:

$$
\begin{aligned}
& \operatorname{Disjoint}\left(A, x, y, \alpha, \alpha^{\prime}\right):= \\
& \quad \operatorname{Path}(A, x, y, \alpha) \wedge \operatorname{Path}\left(A, x, y, \alpha^{\prime}\right) \\
& \quad \wedge(\forall i \leq n-2 \forall j \leq n-2)\left(\alpha(i, j) \cdot \alpha^{\prime}(i, j)=0\right)
\end{aligned}
$$

We leave stating that $x, y$ is a restricted pair to the reader.

We must be able to talk about a collection of paths; the $0-1$ matrix $\beta$ will encode a collection of paths $\alpha_{1}, \alpha_{2}, \ldots, \alpha_{\lambda}$ :

$$
\beta=\begin{array}{|l|l|l|l|}
\hline \beta[1]=\alpha_{1} & \beta[2]=\alpha_{2} & \ldots & \beta[\lambda]=\alpha_{\lambda} \\
\hline
\end{array}
$$

so that $\beta$ is a matrix of size $(n-2) \times \lambda(n-2)$. Each $\beta[i]$ can be defined thus:

$$
\beta[i]:=\lambda_{p q}\langle n-2, n-2, \beta(p,(i-1)(n-2)+q)\rangle .
$$


We are interested in pairwise disjoint collections of paths:

$$
\begin{aligned}
& \operatorname{CollectDisj}(A, x, y, \beta, \lambda):= \\
& \forall i \leq \lambda \operatorname{Path}(A, x, y, \beta[i]) \wedge \\
& (\forall i \neq j \leq \lambda) \operatorname{Disjoint}(A, x, y, \beta[i], \beta[j])
\end{aligned}
$$

The following formula expresses $\lambda(x, y)$ for a given $A$; note that it is a $\Pi_{2}^{B}$ formula:

$$
\begin{aligned}
& \operatorname{MaxDisj}(A, x, y, \lambda):= \\
& (\exists \beta \leq(n-2) \lambda) \operatorname{CollectDisj}(A, x, y, \beta, \lambda) \wedge \\
& (\forall \alpha \leq n-2)(\operatorname{Path}(A, x, y, \alpha) \rightarrow \exists i \leq \lambda \alpha=\beta[i])
\end{aligned}
$$

Likewise, we need to formalize $\kappa(x, y)$; we start with a $0-1$ matrix $\gamma$ expressing a cut in $A$ :

$$
\begin{aligned}
& \operatorname{Cut}(A, \gamma):= \\
& (\forall i \leq n-2)(\forall j \leq n-2)(\gamma(i, j)=1 \rightarrow A(i, j)=1)
\end{aligned}
$$

which says that every edge of $\gamma$ is an edge of $A$, and it defines the cut implicitly as the set of edges in $A$ but no in $\gamma$. Now, the following $\Sigma_{2}^{B}$ formula expresses that there is an $x, y$-cut of size $\kappa$ in $A$ :

$$
\begin{aligned}
& \operatorname{CutSize}(A, x, y, \kappa):= \\
& \exists \gamma \leq(n-2) \operatorname{Cut}(A, \gamma) \wedge \Sigma \gamma=\kappa \wedge(\forall \alpha \leq n-2) \\
& \neg \operatorname{Path}\left(\lambda_{p q}\langle n-2, n-2, A(p, q)-\gamma(p, q)\rangle, x, y, \alpha\right),
\end{aligned}
$$

and the minimum number of edges in an $x, y$-cut can be expressed with a formula that is a conjunction of a $\Sigma_{2}^{B}$ formula with a $\Pi_{2}^{B}$ formula, yielding therefore a formula in $\Sigma_{3}^{B} \cap \Pi_{3}^{B}$ :

$$
\begin{aligned}
& \operatorname{MinCut}(A, x, y, \kappa):= \\
& \operatorname{CutSize}(A, x, y, \kappa) \wedge \neg \operatorname{CutSize}(A, x, y, \kappa-1)
\end{aligned}
$$

Putting it all together, we can state Menger's theorem in $\mathcal{L}_{\mathbf{L A}}$ with a $\Sigma_{3}^{B} \cap \Pi_{3}^{B}$ formula as follows:

$$
\begin{aligned}
& \operatorname{Menger}(A):= \\
& \operatorname{MaxDisj}(A, x, y, \lambda) \wedge \operatorname{MinCut}(A, x, y, \kappa) \rightarrow \lambda=\kappa
\end{aligned}
$$

(Note that if a formula is in $\Sigma_{3}^{B} \cap \Pi_{3}^{B}$, then its negation is still in $\Sigma_{3}^{B} \cap \Pi_{3}^{B}$.)

Let Menger' be the restricted version of Menger's Theorem, i.e., one where $x, y$ is a restricted pair, as in Definition 10. We can now state the main result of this section.

Lemma 11: LA $\cup$ Menger' $^{\prime} \vdash \mathrm{KMM}$.

Proof: Note that the implication resembles the statement of KMM, but the difference is that in KMM the antecedent is a conjunction of two $\Pi_{1}^{B}$ formulas (and hence it is a $\Pi_{1}^{B}$ formula), whereas in Menger's theorem, the antecedent is a $\Sigma_{3}^{B} \cap \Pi_{3}^{B}$ formula.

Suppose that we have $\operatorname{Min} \operatorname{Cover}(A, \alpha) \wedge \operatorname{MaxSelect}(A, \beta)$, the antecedent of KMM (see 5). Using Menger's theorem (see 19) we want to conclude that $\Sigma \alpha=\Sigma \beta$. We do so by restating "covers and selections" of $A$ as "cuts and paths" of a related matrix $A^{\prime}$ defined as: $A^{\prime}$ is a $0-1$ matrix of size $|A|+1$, with entries:

$$
A^{\prime}(i, j)= \begin{cases}A(i, j) & \text { for } 1 \leq i, j \leq|A| \\ 1 & \text { one of }\{i, j\} \text { equals }|A|+1 \\ 0 & \text { both of }\{i, j\} \text { equal }|A|+1\end{cases}
$$

Note that $A^{\prime}$ can be stated succinctly as a term of $\mathcal{L}_{\mathbf{L A}}$ :

$$
\begin{aligned}
& A^{\prime}:=\lambda i j\langle r(A)+1, c(A)+1, \\
& \operatorname{cond}(1 \leq i, j \leq|A|, A(i, j), \operatorname{cond}(i=j=|A|+1,0,1))\rangle
\end{aligned}
$$

The point is that when we view $A$ as representing a bipartite graph (with rows representing $V_{1}$ and columns representing $V_{2}$ and $A(i, j)=1$ iff there is an edge $\left.(i, j) \in V_{1} \times V_{2}\right)$, then $A^{\prime}$ represents a graph where two more vertices are added $(x=|A|+1$ and $y=|A|+1$, the first row and column of $A$, resp.) and $x$ is connected to every vertex in $V_{2}$ and $y$ is connected to every vertex in $V_{1}$, and $x, y$ are not connected to any other vertices.

Also, a maximal selection in $A$ corresponds to a maximal matching in the related graph, and a minimal cover in $A$ corresponds to a minimal cover in the related graph (recall that a cover in a graph is a subset of vertices so that every edge has at least one end-point in this subset). Furthermore, a maximal matching in the graph related to $A$ corresponds to a maximal subset of internally disjoint paths in the graph related to $A^{\prime}$; similarly, a minimal cover in the graph related to $A$ corresponds to a minimal cut in the graph related to $A^{\prime}$.

Finally, let:

$$
A^{\prime \prime}=\left[\begin{array}{cc}
0 & A^{\prime} \\
\left(A^{\prime}\right)^{T} & 0
\end{array}\right]
$$

that is, $A^{\prime \prime}$ is the adjacency matrix of the graph related to $A^{\prime}$ viewed as a normal graph (i.e., not bipartite).

Claim 12: LA proves the following:

- $\operatorname{Min} \operatorname{Cover}(A, \alpha) \leftrightarrow \operatorname{MinCut}\left(A^{\prime \prime}, x, y, \Sigma \alpha\right)$

- $\operatorname{MaxSelect}(A, \beta) \leftrightarrow \operatorname{MaxDisj}\left(A^{\prime \prime}, x, y, \Sigma \beta\right)$

The proof of Claim 12 does not require induction and we leave it to the reader. By Menger's Theorem it follows directly that $\Sigma \alpha=\Sigma \beta$ which also finishes the proof of KMM.

It is easy to check that $x, y$ is a restricted pair (Definition 10 ) in the graph related to $A^{\prime \prime}$.

Lemma 13: LA $\cup \mathrm{KMM} \vdash$ Menger'.

Proof: Suppose that we have $\operatorname{MaxDisj}(A, x, y, \lambda)$ and $\operatorname{MinCut}(A, x, y, \kappa)$; these two formulas assert the existence of $\beta$, a collection of $\lambda$ many pairwise disjoint $x, y$-paths, and $\gamma$, an $x, y$-cut of size $\kappa$. (The constructions of $\beta$ and $\gamma$ have been shown earlier in this section.) We assume that $x, y$ is a restricted pair of vertices, as in Definition 10.

Each path in $\beta$ must have at least one edge in the cut $\gamma$ and no edge of $\gamma$ can be in more than one path in $\beta$, hence $\lambda \leq \kappa$. The proof of this is identical to the proof of Claim 6 .

Thus, it remains to show, using KMM, that $\lambda \geq \kappa$. To this end we proceed as follows: we construct a new matrix $A^{\prime}$, such that each row of $A^{\prime}$ corresponds to one of the paths in $\beta$. Note that since the paths in $\beta$ are disjoint, the number of rows of $A^{\prime}$ is polynomial in the size of $A$; this is a key observation - there can be at most linearly many (in the number of vertices) disjoint paths in a given graph. On the other hand, the columns of $A^{\prime}$ correspond to the edges in $\gamma$, again bounded by a polynomial in the size of $A$, as there are at most $|A|^{2}$ edges in the graph. We have $A^{\prime}(i, j)=1 \Longleftrightarrow$ edge $j$ is in the path $i$. 
Note that $\beta$ and $\gamma$ are built independently; the only assertion we make about their relationship is that they are of the same size, i.e., $\lambda=\kappa$. In the next Claim we show how we can modify $\beta$ and $\gamma$ (using an algorithm provably correct in LA) in order to obtain an $A^{\prime}$ to which we can apply KMM.

Claim 14: We can modify $\beta$ and $\gamma$, with a procedure provably correct in $\mathbf{L A}$, so that each row and column of $A^{\prime}$ contains exactly one 1 .

Before we prove Claim 14 we show how we use it to show that $\lambda \geq \kappa$ : if each row and column of $A^{\prime}$ has exactly one 1 , then $\kappa=o_{A^{\prime}}$, and by KMM, $o_{A^{\prime}}=l_{A^{\prime}} \leq r\left(A^{\prime}\right)=\lambda$.

We now prove Claim 14. First observe that no matter what $\beta$ and $\gamma$ we pick, each column of $A^{\prime}$ has at most one 1 , and each row of $A^{\prime}$ has at least one 1 . The reason is that the paths in $\beta$ are pairwise disjoint - hence they never share an edge. If a row of $A^{\prime}$ contains no $1 \mathrm{~s}$, then we have an $x, y$-path, and $\gamma$ is not an $x, y$-cut.

Suppose there is a column without a 1 . Then there is an edge $e \in \gamma$ that does not belong to any path in $\beta$; if $e$ were unnecessary, we could lower $\kappa$, and get a contradiction (with the minimality of $\kappa$ ). Thus, $e$ cuts some $x, y$-path $\rho$ not in $\beta$; by the maximality of $\beta, \rho$ shares an edge $e^{\prime}$ with some path $\rho^{\prime}$ which is in $\beta$. If $e^{\prime}$ is in the cut $\gamma$, then $e$ is not needed contradiction again. So $e^{\prime}$ is not in $\gamma$; exchange $e$ and $e^{\prime}$ in order to obtain a new $\gamma$. Here is were we use the restriction on the graphs, i.e., we know that $e$ is not shared by any other paths, as $\rho$ shares its edge with $\rho^{\prime}$. We leave showing that each row has at most one 1 to the reader.

\section{B. Hall's Theorem}

Let $S_{1}, S_{2}, \ldots, S_{n}$ be $n$ subsets of a given set $M$. Let $D$ be a set of $n$ elements of $M, D=\left\{a_{1}, a_{2}, \ldots, a_{n}\right\}$, such that $a_{i} \in S_{i}$ for each $i=1,2, \ldots, n$. Then $D$ is said to be a system of distinct representative (SDR) for the subsets $S_{1}, S_{2}, \ldots, S_{n}$.

If the subsets $S_{1}, S_{2}, \ldots, S_{n}$ have an SDR, then any $k$ of the sets must contain between them at least $k$ elements. The converse proposition is the combinatorial theorem of P. Hall: suppose that for any $k=1,2, \ldots, n$, any $S_{i_{1}} \cup S_{i_{2}} \cup \cdots \cup S_{i_{k}}$ contains at least $k$ elements of $M$; we call this the union property. Then there exists an SDR for these subsets. (See [14], [15], [16] for more on Hall's theorem.)

We formalize Hall's theorem in $\mathcal{L}_{\mathbf{L A}}$ with an adjacency matrix $A$ such that the rows of $A$ represent the sets $S_{i}$, and the columns of $A$ represent the indices of the elements in $M$, i.e., the columns are labeled with $[n]=\{1,2, \ldots, n\}$, and $A(i, j)=1 \Longleftrightarrow j \in S_{i}$. Let $\operatorname{SDR}(A)$ be the following $\Sigma_{1}^{B}$ formula which states that $A$ has a system of distinct representatives:

$$
\operatorname{SDR}(A):=(\exists P \leq n)(\forall i \leq n)(A P)_{i i}=1
$$

We reserve the letters $P, Q$ for permutation matrices, and $(\exists P \leq n) \phi$ abbreviates $(\exists P)[\operatorname{Perm}(P) \wedge|P| \leq n \wedge \phi]$ (similarly for $(\forall P \leq n) \phi$, but with an implication instead of a conjunction), where Perm is a $\Sigma_{0}^{B}$ predicate stating that $P$ is a permutation matrix (a unique 1 in each row and column). See [8] for more details about handling permutation matrices.
The next predicate is a $\Pi_{2}^{B}$ formula stating the union property:

$$
\begin{aligned}
& \text { UnionProp }(A):= \\
& \forall P \leq n \forall k \leq n \exists Q \leq n \\
& {\left[\forall i \leq k\left(\lambda_{p q}\left\langle k, 1,(P A Q)_{p i}\right\rangle \neq \lambda_{p q}\langle k, 1,0\rangle\right)\right]}
\end{aligned}
$$

Therefore, we can state Hall's theorem as a $\Sigma_{2}^{B}$ formula:

$$
\operatorname{Hall}(A):=\operatorname{UnionProp}(A) \rightarrow \operatorname{SDR}(A)
$$

Lemma 15: LA $\cup \mathrm{KMM} \vdash$ Hall.

Proof: Let $A$ be a 0-1 sets/elements incidence matrix of size $n \times n$. Assume that we have $\operatorname{UnionProp}(A)$; our goal is to show in $\mathbf{L A}$, using $\mathrm{KMM}$, that $\operatorname{SDR}(A)$ holds.

Since by Claim 4 , every matrix can be put in a diagonal form, using the fact that we have $\operatorname{UnionProp}(A)$, it follows that we can find $P, Q \leq n$ such that $\forall k \leq n(P A Q)_{k k}=1$. Thus we need $n$ lines to cover all the $1 \mathrm{~s}$, but by KMM there exists a selection of $n 1 \mathrm{~s}$ no two on the same line, hence, $A$ is of term rank $n$.

But this means that the maximal selection of $1 \mathrm{~s}$, no two on the same line, constitutes a permutation matrix $P$ (since $A$ is $n \times n$, and we have $n 1 \mathrm{~s}$, no two on the same line). Note that $A P^{T}$ has all ones on the diagonal, and this in turn implies $\operatorname{SDR}(A)$.

\section{Lemma 16: LA $\cup$ Hall $\vdash$ KMM.}

Proof: Suppose that we have $\operatorname{Min} \operatorname{Cover}(A, \alpha)$ and $\operatorname{Max} \operatorname{Select}(A, \beta)$; we want to conclude that $\Sigma \alpha=\Sigma \beta$ using Hall's Theorem.

As usual, let $l_{A}=\Sigma \alpha$ and $o_{A}=\Sigma \beta$, and by Claim 6 we already have that $\mathbf{L A} \vdash o_{A} \leq l_{A}$ (see Section ஹ). We now show in LA that $o_{A} \geq l_{A}$ using Hall's Theorem.

Suppose that the minimum number of lines that cover all the 1s of $A$ consists of $e$ rows and $f$ columns, so that $l_{A}=e+f$. Both $l_{A}$ and $o_{A}$ are invariant under permutations of the rows and the columns of $A$ (Lemma 2), and so we reorder the rows and columns of $A$ so that these $e$ rows and $f$ columns are the initial rows and columns of $A^{\prime}$,

$$
A^{\prime}=\left[\begin{array}{ll}
A_{1} & A_{2} \\
A_{3} & A_{4}
\end{array}\right],
$$

where $A_{1}$ is of size $e \times f$. Now, we shall work with the term rank of $A_{2}$ and $A_{3}$ in order to show that $o_{A} \geq l_{A}$. More precisely, we will show that the maximum number of $1 \mathrm{~s}$, no two on the same line, in $A_{2}$ is $e$, while in $A_{3}$ it is $f$.

Let us consider $A_{2}$ as an incidence matrix for subsets $S_{1}, S_{2}, \ldots, S_{e}$ of a universe of size $|A|-f$, and $A_{3}^{t}$ (which is the transpose of $A_{3}$ ) as an incidence matrix for subsets $S_{1}^{\prime}, S_{2}^{\prime}, \ldots, S_{f}^{\prime}$ of a universe of size $|A|-e$. It is not difficult to prove that $\operatorname{UnionProp}\left(A_{2}\right)$ and $\operatorname{Union} \operatorname{Prop}\left(A_{3}^{t}\right)$ holds (and can be proven in $\mathbf{L A}$; this is left to the reader), which in turn implies $\operatorname{SDR}\left(A_{2}\right)$ and $\operatorname{SDR}\left(A_{3}^{t}\right)$, resp., by Hall's Theorem. But the system of distinct representative of $A_{2}$ (resp. $A_{3}^{t}$ ) implies that $o_{A_{2}} \geq e$ (resp. $o_{A_{3}^{t}}=o_{A_{3}} \geq f$ ), and since $o_{A} \geq o_{A_{2}}+o_{A_{3}}$, this yields that $o_{A} \geq e+f=l_{A}$. 


\section{Dilworth's Theorem}

Let $\mathcal{P}$ be a finite partially ordered set or poset (we use a "script $\mathcal{P}$ " in order to distinguish it from permutation matrices, denoted with $P$ ). We say that $a, b \in \mathcal{P}$ are comparable elements if either $a<b$ or $b<a$. A subset $C$ of $\mathcal{P}$ is a chain if any two distinct elements of $C$ are comparable. A subset $S$ of $\mathcal{P}$ is an anti-chain (also called an independent set) if no two elements of $S$ are comparable.

We want to partition a poset into chains; a poset with an anti-chain of size $k$ cannot be partitioned into fewer than $k$ chains, because any two elements of the anti-chain must be in a different partition. Dilworth's Theorem states that the maximum size of an anti-chain equals the minimum number of chains needed to partition $\mathcal{P}$. (For more on Dilworth's Theorem see [17], [18]).

In order to formalize Dilworth's theorem in $\mathcal{L}_{\mathbf{L A}}$, we represent finite posets $\mathcal{P}=\left(X=\left\{x_{1}, x_{2}, \ldots, x_{n}\right\},<\right)$ with an incidence matrix $A=A_{\mathcal{P}}$ of size $|X| \times|X|$, which expresses the relation $<$ as follows: $A(i, j)=1 \Longleftrightarrow x_{i}<x_{j}$. For more material regarding formalizing posets see [19].

We let a $1 \times n$ matrix $\alpha$ encode a chain as follows:

$$
\begin{aligned}
\operatorname{Chain}(A, \alpha) & :=(\forall i \neq j \leq n) \\
& {[\alpha(i)=\alpha(j)=1 \rightarrow A(i, j)=1 \vee A(j, i)=1] . }
\end{aligned}
$$

In a similar fashion to 23 we define an anti-chain $\gamma$; the only difference is that the succedent of the implication expresses the opposite: $A(i, j)=0 \wedge A(j, i)=0$.

Recall that using (13) we were able to talk about a collection of paths; in a similar vain, we can use $\mathcal{L}_{\mathrm{LA}}$ to talk about a collection of chains of $\mathcal{P}$ : $\beta$ is an $1 \times \kappa \cdot n$ matrix which encodes the contents of $\kappa$ many chains. We can then talk about a minimal collection of chains, or a maximal size of an antichain in the usual fashion. Since we have done this already for collections of paths, we omit the details in the interest of space. The reader is encouraged to fill in the details.

We can state Dilworth's Theorem as follows:

$$
\begin{aligned}
& \operatorname{Dilworth}(A):=\left(\exists \beta \leq|A|^{2}\right)(\exists \gamma \leq|A|) \\
& \quad \operatorname{MinChain}(A, \beta, \kappa) \wedge \operatorname{Max} \operatorname{AntiChain}(A, \gamma, \lambda) \rightarrow \lambda=\kappa
\end{aligned}
$$

where the predicate $\operatorname{MinChain}(A, \beta, \kappa)$ asserts that $\beta$ is a collection of $\kappa$ many chains that partition the poset, and the predicate $\operatorname{MaxAntiChain}(A, \gamma, \lambda)$ asserts that $\gamma$ is an antichain consisting of $\lambda$ elements. Again, the details of the $\mathcal{L}_{\mathbf{L A}}$ definitions can be provided by the reader, in light of the definitions given in Section $\mathrm{V}-\mathrm{A}$

Lemma 17: LA $\cup$ KMM $\vdash$ Dilworth

Proof: Suppose that we have $\operatorname{MinChain}(A, \beta, \kappa)$ and $\operatorname{Max} \operatorname{AntiChain}(A, \gamma, \lambda)$; we want to use $\mathbf{L A}$ reasoning and KMM in order to show that $\lambda=\kappa$.

As usual we define a matrix $A^{\prime}$ whose rows are labeled by the chains in $\beta$, and whose columns are labeled by the elements of the poset. As there cannot be more chains than elements in the poset, it follows that the number of rows of $A^{\prime}$ is bounded by $|A|$ (while the number of columns is exactly $|A|$ ). The proof of this is similar to the proof of Claim 6 .

We have that $A^{\prime}(i, j)=1 \Longleftrightarrow$ chain $i$ contains element $j$. Clearly each column contains at least one 1 , as $\beta$ is a partition of the poset. On the other hand, rows may contain more than one 1, as in general chains may have more than one element.

Note that a maximal selection of $1 \mathrm{~s}$, no two of them on the same line, corresponds naturally to a maximal anti-chain; such a selection picks one 1 from each line, and so its size is the number of rows of $A^{\prime}$. By KMM, it follows that

$$
\lambda=o_{A^{\prime}}=l_{A^{\prime}}=r\left(A^{\prime}\right)=\kappa,
$$

where $r\left(A^{\prime}\right)$ is the number of rows of $A^{\prime}$.

Lemma 18: LA $\cup$ Dilworth $\vdash \mathrm{KMM}$

Proof: It is in fact easier to show that that $\mathbf{L A} \cup$ Dilworth $\vdash$ Hall, and since by Lemma 16 we have that $\mathbf{L A} \cup$ Hall $\vdash \mathrm{KMM}$, we will be done.

In order to prove Hall using Dilworth and LA reasoning, we assume that we have $A$, a $0-1$ sets/elements incidence matrix of size $n \times n$. Assume that we have $\operatorname{UnionProp}(A)$; our goal is to show in $\mathbf{L A}$, using Dilworth, that $\operatorname{SDR}(A)$ holds.

Let $S_{1}, S_{2}, \ldots, S_{n}$ be subsets of $\left\{x_{1}, x_{2}, \ldots, x_{n}\right\}$ where $n=|A|$. We define a partial order $\mathcal{P}$ based on $A$; the universe of $\mathcal{P}$ is $X=\left\{S_{1}, S_{2}, \ldots, S_{n}\right\} \cup\left\{x_{1}, \ldots, x_{n}\right\}$. The relation $<_{\mathcal{P}}$ is defined as follows: $x_{i}<_{\mathcal{P}} S_{j} \Longleftrightarrow A(i, j)=1$.

Claim 19: The maximum size of an anti-chain in $\mathcal{P}$ is $n$.

Proof: The $\left\{x_{1}, \ldots, x_{n}\right\}$ form an anti-chain of length $n$, and we cannot add any of the $S_{j}$, as some $x_{i} \in S_{j}$, and hence $x_{i}<\mathcal{P} S_{j}$.

By Dilworth we can partition $\mathcal{P}$ into $n$ chains, where each of the chains has two elements $\left\{x_{i}, S_{j}\right\}$, giving us the set of distinct representatives, and hence $\operatorname{SDR}(A)$.

\section{FUTURE WORK}

The main open question is the following: is KMM equivalent (in LA) to the general version of Menger's Theorem? That is, can we lift the restriction that $x, y$ is a restricted pair of vertices (Definition 10). There are many proofs of the general version of Menger's theorem - for example [12] is clearly formalizable in $\exists \mathbf{L A}$. But it would be very interesting to know whether the general version of Menger's Theorem is equivalent to $\mathrm{KMM}$ in low complexity.

Now that we know that $\exists \mathbf{L A} \vdash$ KMM (Theorem 1) and that $\mathrm{KMM}$ is equivalent to a host of other combinatorial theorems - and this equivalence can be shown in the weak theory LA (Theorem 9) - it would be interesting to know whether it is also the case that:

$$
\text { - } \mathbf{L A} \cup \mathrm{KMM} \vdash \mathrm{PHP}
$$$$
\text { - } \mathbf{L A} \cup \mathrm{PHP} \vdash \mathrm{KMM}
$$

that is, whether $\mathbf{L A}$ can prove the equivalence of KMM and the pigeonhole principle. We conjecture that the first assertion is true, and that it should not be too difficult to show it. The second assertion is probably not true. Note that in the proof of Claim 6 we implicitly show a certain weaker kind of the PHP in LA: we showed that if we have a set of $n$ items $\left\{i_{1}, i_{2}, \ldots, i_{n}\right\}$ and a second set of $m$ items $\left\{j_{1}, j_{2}, \ldots, j_{m}\right\}$, 
and we can match each $i_{p}$ with some $j_{q}$, and this matching is both definable in $\mathbf{L A}$ and its injectivity is provable in $\mathbf{L A}$, then $n \leq m$. We did this by defining an incidence matrix $A$ such that $A(p, q)=1 \Longleftrightarrow i_{p} \mapsto j_{q}$. If this mapping is injective, then each column of $A$ has at most one 1; thus:

$$
n \leq \Sigma A=\Sigma_{i}(\operatorname{col} i \text { of } A) \leq \Sigma_{i} 1 \leq m .
$$

Also, we would like to know whether $\mathbf{L A} \cup \mathrm{KMM}$ can prove hard matrix identities, such as $A B=I \rightarrow B A=I$. Of course, we already know from [4] that (non-uniform) $\mathbf{N C}^{2}$ Frege is sufficient to prove $A B=I \rightarrow B A=I$. On the other hand, is it possible that $\mathbf{L A}$ together with $A B=I \rightarrow B A=I$ can prove KMM? This would imply that $A B=I \rightarrow B A=I$ is "complete" for combinatorial matrix algebra, in the sense that all of combinatorial matrix algebra follows from this principle with proofs of low complexity.

Furthermore, given two 0-1 matrices $A, B$, what can we say about $l_{A B}$ and $o_{A B}$ ? From Claim 2 we know that if $B$ is a permutation matrix, then $l_{A B}=l_{A}$ and $o_{A B}=o_{A}$ (and similarly, if $A$ is a permutation matrix); but what can be said in general? Of course, the understanding here is that multiplication is over the field $\{0,1\}$.

\section{APPENDIX - LA}

The logical theory $\mathbf{L A}$ is strong enough to prove all the ring properties of matrices such as $A(B C)=(A B) C$ and $A+B=B+A$, but weak enough so that the theorems of LA translate into propositional tautologies with short Frege proofs. LA has three sorts of object: indices (i.e., natural numbers), ring elements, and matrices, where the corresponding variables are denoted $i, j, k, \ldots ; a, b, c, \ldots$; and $A, B, C, \ldots$, respectively. The semantic assumes that objects of type ring are from a fixed but arbitrary ring (for the purpose of this paper we are only interested in the ring $\mathbb{Z}$ ), and objects of type matrix have entries from that ring.

Terms and formulas are built from the following function and predicate symbols, which together comprise the language $\mathcal{L}_{\text {LA }}$ :

$$
\begin{aligned}
& 0_{\text {index }}, 1_{\text {index }},+_{\text {index }}, *_{\text {index }},-_{\text {index }}, \text { div }, \text { rem, } \\
& 0_{\text {ring }}, 1_{\text {ring }},+_{\text {ring }}, *_{\text {ring }},-_{\text {ring }},{ }^{-1}, r, \mathrm{c}, \mathrm{e}, \Sigma, \\
& \leq_{\text {index }},=_{\text {index }},=_{\text {ring }},=_{\text {matrix }}, \text { cond }_{\text {index }}, \text { cond }_{\text {ring }}
\end{aligned}
$$

The intended meaning should be clear, except in the case of ${ }_{-}{ }_{\text {index }}$, cut-off subtraction, defined as $i-j=0$ if $i<j$. For a matrix $A$ : $\mathrm{r}(A), \mathrm{c}(A)$ are the numbers of rows and columns in $A, \mathrm{e}(A, i, j)$ is the ring element $A_{i j}$ (where $A_{i j}=0$ if $i=0$ or $j=0$ or $i>\mathrm{r}(A)$ or $j>\mathrm{C}(A)), \Sigma(A)$ is the sum of the elements in $A$. Also $\operatorname{cond}\left(\alpha, t_{1}, t_{2}\right)$ is interpreted if $\alpha$ then $t_{1}$ else $t_{2}$, where $\alpha$ is a formula all of whose atomic sub-formulas have the form $m \leq n$ or $m=n$, where $m, n$ are terms of type index, and $t_{1}, t_{2}$ are terms either both of type index or both of type ring. The subscripts index, ring, and matrix are usually omitted, since they ought to be clear from the context.

We use $n, m$ for terms of type index, $t, u$ for terms of type ring, and $T, U$ for terms of type matrix. Terms of all three types are constructed from variables and the symbols above in the usual way, except that terms of type matrix are either variables $A, B, C, \ldots$ or $\lambda$-terms $\lambda i j\langle m, n, t\rangle$. Here $i$ and $j$ are variables of type index bound by the $\lambda$ operator, intended to range over the rows and columns of the matrix. Also $m, n$ are terms of type index not containing $i, j$ (representing the numbers of rows and columns of the matrix) and $t$ is a term of type ring (representing the matrix element in position $(i, j)$ ).

Atomic formulas are of the form $m \leq n, m=n, t=u$ and $T=U$, where the three occurrences of = formally have subscripts index, ring, matrix, respectively. General formulas are built from atomic formulas using the propositional connectives $\neg, \vee, \wedge$ and quantifiers $\forall, \exists$.

\section{A. Axioms and rules of $\mathbf{L A}$}

For each axiom listed below, every legal substitution of terms for free variables is an axiom of $\mathbf{L A}$. Note that in a $\lambda$ term $\lambda i j\langle m, n, t\rangle$ the variables $i, j$ are bound. Substitution instances must respect the usual rules which prevent free variables from being caught by the binding operator $\lambda i j$. The bound variables $i, j$ may be renamed to any new distinct pair of variables.

1) Equality Axioms: These are the usual equality axioms, generalized to apply to the three-sorted theory LA. Here = can be any of the three equality symbols, $x, y, z$ are variables of any of the three sorts (as long as the formulas are syntactically correct). In A4, the symbol $f$ can be any of the non-constant function symbols of LA. However A5 applies only to $\leq$, since this in the only predicate symbol of LA other than $=$.

$$
\begin{array}{ll}
\text { A1 } & x=x \\
\text { A2 } & x=y \rightarrow y=x \\
\text { A3 } & (x=y \wedge y=z) \rightarrow x=z \\
\text { A4 } & x_{1}=y_{1}, \ldots, x_{n}=y_{n} \rightarrow f x_{1} \ldots x_{n}=f y_{1} \ldots y_{n} \\
\text { A5 } & i_{1}=j_{1}, i_{2}=j_{2}, i_{1} \leq i_{2} \rightarrow j_{1} \leq j_{2}
\end{array}
$$

2) Axioms for indices: These are the axioms that govern the behavior of index elements. The index elements are used to access the entries of matrices, and so we need to define some basic number theoretic operations.

$$
\begin{aligned}
& \text { A6 } i+1 \neq 0 \\
& \text { A7 } i *(j+1)=(i * j)+i \\
& \text { A8 } i+1=j+1 \rightarrow i=j \\
& \text { A9 } i \leq i+j \\
& \text { A10 } i+0=i \\
& \text { A11 } i \leq j \wedge j \leq i \\
& \text { A12 } i+(j+1)=(i+j)+1 \\
& \text { A13 }[i \leq j \wedge j \leq i] \rightarrow i=j \\
& \text { A14 } i * 0=0 \\
& \text { A15 }[i \leq j \wedge i+k=j] \rightarrow j-i=k \\
& \text { A16 } \neg(i \leq j) \rightarrow j-i=0 \\
& \text { A17 }[\alpha \rightarrow \operatorname{cond}(\alpha, i, j)=i] \wedge[\neg \alpha \rightarrow \operatorname{cond}(\alpha, i, j)=j]
\end{aligned}
$$

3) Axioms for a ring: These are the axioms that govern the behavior for ring elements; addition and multiplication, as well as additive inverses. We do not need multiplicative inverses.

A18 $0 \neq 1 \wedge a+0=a$ 


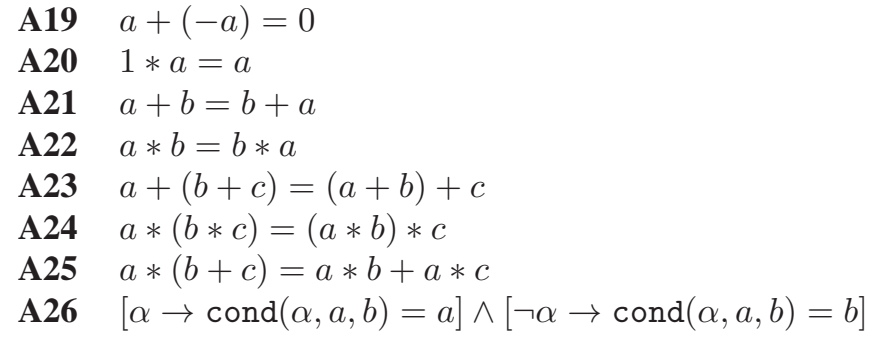

4) Axioms for matrices: Axiom A27 states that $\mathrm{e}(A, i, j)$ is zero when $i, j$ are outside the size of $A$. Axiom A28 defines the behavior of constructed matrices. Axioms A29-A32 define the function $\Sigma$ recursively by first defining it for row vectors, then column vectors $\left(A^{t}:=\lambda i j\left\langle\mathrm{c}(A), \mathrm{r}(A), A_{j i}\right\rangle\right)$, and then in general using the decomposition (26). Finally, axiom A33 takes care of empty matrices.

$$
\begin{aligned}
& \text { A27 }(i=0 \vee r(A)<i \vee j=0 \vee \mathrm{c}(A)<j) \rightarrow \\
& \rightarrow \mathrm{e}(A, i, j)=0 \\
& \text { A28 } r(\lambda i j\langle m, n, t\rangle)=m \wedge \mathrm{c}(\lambda i j\langle m, n, t\rangle)=n \wedge \\
& {[1 \leq i \wedge i \leq m \wedge 1 \leq j \wedge j \leq n] \rightarrow} \\
& \rightarrow \mathrm{e}(\lambda i j\langle m, n, t\rangle, i, j)=t \\
& \text { A29 } \mathrm{r}(A)=1, \mathrm{c}(A)=1 \rightarrow \Sigma(A)=\mathrm{e}(A, 1,1) \\
& \text { A30 } r(A)=1 \wedge 1<\mathrm{c}(A) \rightarrow \Sigma(A)= \\
& =\Sigma\left(\lambda i j\left\langle 1, \mathrm{C}(A)-1, A_{i j}\right\rangle\right)+A_{1 \mathrm{c}(A)} \\
& \text { A31 } \mathrm{c}(A)=1 \rightarrow \Sigma(A)=\Sigma\left(A^{t}\right) \\
& \text { A32 } 1<r(A) \wedge 1<\mathrm{C}(A) \rightarrow \Sigma(A)= \\
& =\mathrm{e}(A, 1,1)+\Sigma(\mathrm{R}(A))+\Sigma(\mathrm{S}(A))+\Sigma(\mathrm{M}(A)) \\
& \text { A33 } r(A)=0 \vee \mathrm{c}(A)=0 \rightarrow \Sigma A=0 \\
& \mathrm{R}(A):=\lambda i j\langle 1, \mathrm{c}(A)-1, \mathrm{e}(A, 1, i+1)\rangle, \\
& \mathrm{S}(A):=\lambda i j\langle\mathrm{r}(A)-1,1, \mathrm{e}(A, i+1,1)\rangle \text {, } \\
& \mathrm{M}(A):=\lambda i j\langle\mathrm{r}(A)-1, \mathrm{c}(A)-1, \mathrm{e}(A, i+1, j+1)\rangle \text {. }
\end{aligned}
$$

5) Rules for LA: In addition to all the axioms just presented, LA has two rules: matrix equality and induction.

\section{Matrix equality rule}

From the premises: $\mathrm{e}(T, i, j)=\mathrm{e}(U, i, j), r(T)=\mathrm{r}(U)$ and $\mathrm{c}(T)=\mathrm{C}(U)$, we conclude $T=U$.

The only restriction is that the variables $i, j$ may not occur free in $T=U$; other than that, $T$ and $U$ can be arbitrary matrix terms. Our semantics implies that $i$ and $j$ are implicitly universally quantified in the top formula. The rule allows us to conclude $T=U$, provided that $T$ and $U$ have the same numbers of rows and columns, and corresponding entries are equal.

Induction rule $\alpha(i) \rightarrow \alpha(i+1)$ implies $\alpha(0) \rightarrow \alpha(n)$.

Here $\alpha(i)$ is any formula, $n$ is any term of type index, and $\alpha(n)$ indicates $n$ is substituted for free occurrences of $i$ in $\alpha(i)$. (Similarly for $\alpha(0)$.) Note that in LA we only allow induction over $\Sigma_{0}^{B}$ formulas (no matrix quantifiers), whereas in $\exists \mathbf{L A}$ we allow induction over $\Sigma_{1}^{B}$ formulas (a single block of bounded existential matrix quantifiers when $\alpha$ is put in prenex form). This completes the description of $\mathbf{L A}$. We finish this section by observing the substitution property in the lemma below. We say that a formula $S^{\prime}$ of $\mathbf{L A}$ is a substitution instance of a formula $S$ of $\mathbf{L A}$ provided that $S^{\prime}$ results by substituting terms for free variables of $S$. Of course each term must have the same sort as the variable it replaces, and bound variables must be renamed as appropriate.

Lemma 20: Every substitution instance of a theorem of $\mathbf{L A}$ is a theorem of $\mathbf{L A}$.

This follows by straightforward induction on $\mathbf{L A}$ proofs. The base case follows from the fact that every substitution instance of an LA axiom is an LA axiom.

\section{REFERENCES}

[1] D. König, "Gráfok és alkalmazásuk a determinánsok és a halmazok elméletére," Matematikai és Természettudományi Értesítö, vol. 34, pp. 104-119, 1916.

[2] — , "Über graphen und ihre anwendung auf determinantentheorie und mengenlehre," Mathematische Annalen, vol. 77, no. 4, 1916.

[3] D. T. M. Lê and S. A. Cook, "Formalizing randomized matching algorithms," Logical Methods in Computer Science, vol. 8, pp. 1-25, 2012.

[4] P. Hrubes and I. Tzameret, "Short proofs for the determinant identities," CoRR, vol. abs/1112.6265, December 2011.

[5] R. A. Brualdi and H. J. Ryser, Combinatorial Matrix Theory. Cambridge University Press, 1991.

[6] M. Soltys and S. Cook, "The complexity of derivations of matrix identities," Annals of Pure and Applied Logic, vol. 130, no. 1-3, pp. 207-275, December 2004.

[7] E. Jeřábek, "Weak pigeonhole principle, and randomized computation," Ph.D. dissertation, Faculty of Mathematics and Physics, Charles University, Prague, 2005.

[8] M. Soltys, "LA, permutations, and the Hajós calculus," Theoretical Computer Science, vol. 348, no. 2-3, pp. 321-333, December 2005.

[9] J. E. Hopcroft and R. M. Karp, "An $n^{5 / 2}$ algorithm for maximum matchings in bipartite graphs," SIAM Journal on Computing, vol. 2, no. 4, December 1973.

[10] M. Soltys and A. Fernandez, "A linear-time algorithm for computing minimum vertex covers from maximum matchings," October 2012, available from www.cas.mcmaster.ca/ soltys.

[11] K. Menger, "Zur allgemeinen kurventheorie," Fund. Math, vol. 10, no. 95-115, 1927.

[12] F. Göring, "Short proof of Menger's theorem," Discrete Mathematics, vol. 219, pp. 295-296, 2000. [Online]. Available: http://www.sciencedirect.com/science/article/pii/S0012365X00000881

[13] J. S. Pym, "A proof of Menger's theorem," Monatshefte für Mathematik, vol. 73, no. 1, pp. 81-83, 1969.

[14] P. Hall, "On representatives of subsets," in Classic Papers in Combinatorics, ser. Modern Birkhäuser Classics, I. Gessel and G.-C. Rota, Eds. Birkhäuser Boston, 1987, pp. 58-62.

[15] C. J. Everett and G. Whaples, "Representations of sequences of sets,' American Journal of Mathematics, vol. 71, no. 2, pp. pp. 287-293, April 1949. [Online]. Available: http://www.jstor.org/stable/2372244

[16] P. R. Halmos and H. E. Vaughan, "The marriage problem," American Journal of Mathematics, vol. 72, no. 1, pp. pp. 214-215, Januar 1950. [Online]. Available: http://www.jstor.org/stable/2372148

[17] R. P. Dilworth, "A decomposition theorem for partially ordered sets," Annals of Mathematics, vol. 51, no. 1, pp. pp. 161-166, January 1950. [Online]. Available: http://www.jstor.org/stable/1969503

[18] M. A. Perles, "A proof of dilworth's decomposition theorem for partially ordered sets," Israel Journal of Mathematics, vol. 1, pp. 105-107, 1963.

[19] M. Soltys, "Feasible proofs of Szpilrajn's theorem: a proof-complexity framework for concurrent automata," Journal of Automata, Languages and Combinatorics (JALC), vol. 16, no. 1, pp. 27-38, 2011. 